\title{
MAR_SECURITY: A JOINT SCHEME FOR IMPROVING THE SECURITY IN VANET USING SECURE GROUP KEY MANAGEMENT AND CRYPTOGRAPHY (SGKC)
}

\author{
Mahabaleshwar Kabbur and V. Arul Kumar \\ School of Computer Science \& Applications, \\ REVA University, Bengaluru-64, Karnataka, India
}

\begin{abstract}
Vehicular Ad-hoc network (VANET) has gained huge attraction from research community due to their significant nature of providing the autonomous vehicular communication. The efficient communication is considered as prime concern in these networks however, several techniques have been introduced to improve the overall communication of VANETs. Security and privacy are also considered as prime aspects of VANETs. Maintaining data security and privacy is highly dynamic VANETs is a challenging task. Several techniques have been introduced recently which are based on the cryptography and key exchange. However, these techniques provide solution to limited security threats. Hence, this work introduces a novel approach for key management and distribution in VANET to provide the security to the network and its components. This approach is later incorporated with cryptography mechanism to secure data packets. Hence, the proposed approach is named as Secure Group Key Management and Cryptography (SGKC). The experimental study shows significant improvements in the network performance. This SGKC approach will help the VANET user's fraternity to perform secured data transmission.
\end{abstract}

\section{KEYWORDS}

Network Protocols, Wireless Network, Mobile Network, Virus, Worms \& Trojan.

\section{INTRODUCTION}

The transportation system plays an important role in the development of any country's economic growth. Thus, the demand for vehicles increases. This increased utilization of vehicles has several advantages such as better and efficient transportation, and also it has several disadvantages related to road safety and other issues such as accidents. A recent study revealed that total 232 billion accidents are reported in the United States and 100 thousand deaths are reported every year in China, and it is still increasing [1]. In these accidents, more than $57 \%$ of accidents are caused due to human error such as lack of attention, poor cooperation among vehicle drivers and poor decisions. The frequent exchange of accident alarm between vehicles can help to avoid these incidents. This communication between vehicles can be performed using wireless communication. Recently, increased the growth of wireless communication has gained huge attraction in various real-time applications such as mobile communication, wireless sensor networks, and satellite communications, etc. 
The technological growth in networking, embedded technology has enabled various development opportunities for the automobile industry due to that vehicles are equipped with various types of smart devices such as Wi-Fi, GPS and other smart devices. Due to these smart devices, vehicles can communicate with each other in wireless manner and facilitates the formation of Vehicular Ad Hoc Network (VANET) where vehicles can communicate to avoid congestion and accidents. Recently, numerous researches are conducted to the establishment of reliable Intelligent Transport System (ITS) which has several facilities such as traffic monitoring, collision control, traffic flow control, nearby location information services, and internet availability in vehicles. Generally, VANETs are characterized by the following factors such as dynamic network topology, on-board sensors, unlimited power, and storage, etc. Similarly, the VANET communication systems can be classified based on the communication types which are: communication inside the vehicle, vehicle to vehicle communication, vehicle to road-sideinfrastructure and hybrid communication V2X where a vehicle can communicate to the vehicle and road-side units [2]. Due to the aforementioned reasons, VANET security is widely studied. These attacks include availability attack such as denial of service, authenticity attacks such as sybil attack, data confidentiality such as eavesdropping, data trust and non-repudiation such as loss of event traceability. Figure 1 shows a classification of various attacks on VANET.

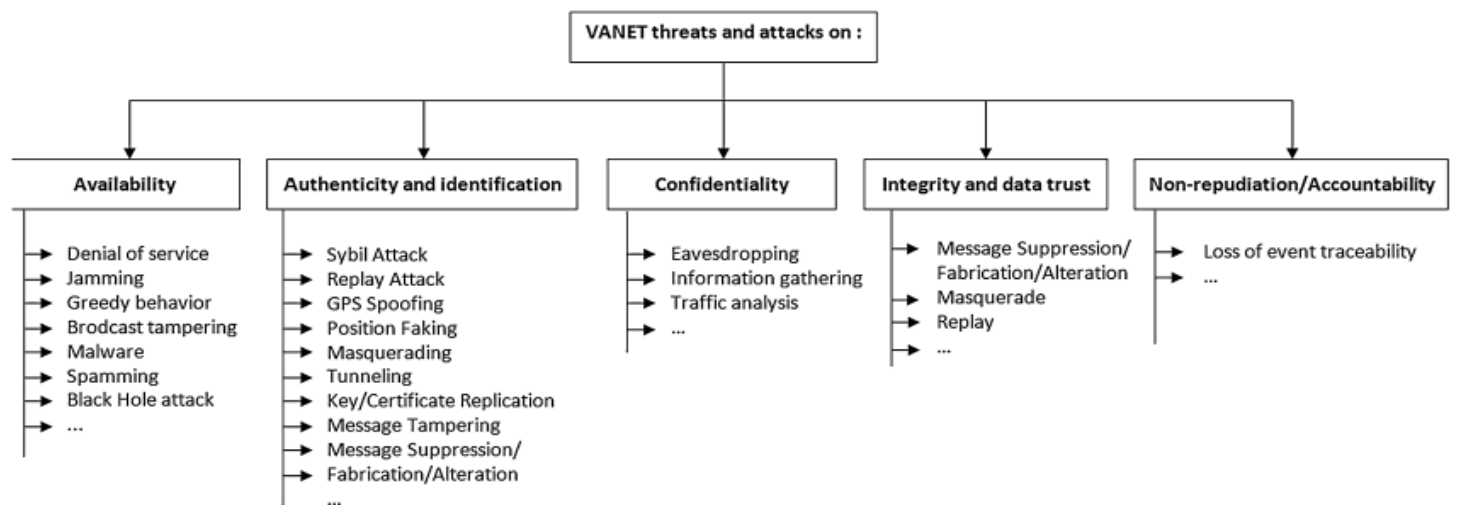

Fig. 1: Examples of VANET threats and attacks

These attacks can lead towards the development of a poor ITS. Hence, security becomes the prime concern for these applications. The Several routing approaches have been introduced which include AODV [3], DSDV [4], DSR [5] and OLSR [6] for efficient data delivery and communication. Moreover, heuristic optimization algorithms are also introduced such as Heuristic algorithm using Ant Colony Optimization [7], Meta-heuristic [8], CACONET [9], and improved hybrid ant particle optimization (IHAPO) [10], etc. Similarly, artificial intelligence schemes are also introduced such as Fuzzy Logic based routing [11], and neural network [12], etc. Several techniques are introduced to overcome the security related issues in VANET. Recently, CARAVAN [13], AMOEBA [14], REP [15], VSPN [16] and many more approaches have been developed to facilitate the location privacy. Similarly, the cryptography based schemes are also introduced to secure the message. [17] Introduced cryptography approach to deal with the Sybil attacks. Some of the security schemes are introduced based on the key-management protocol such as [18] presented Diffie-Hellman key generation scheme. Key management and key generation are the crucial stages of authentication. [19] Presented ID-based authentication protocol. Furthermore, the cryptographic schemes are expanded based on the symmetric and asymmetric cryptography schemes [7]. However, achieving security in these types of dynamic networks is always considered as a challenging task and various researches are still under progress to provide more security in VANETs. This work focuses on security requirement of VANET and introduces a novel approach for secure communication in VANETs. The main contributions of the work are as follows: 
- Development of a novel approach for group key distribution which includes authentication process to improve the network security.

- Incorporating a novel data encryption process based on the Elliptic Curve Cryptography (ECC) scheme.

Rest of the manuscript is organized as: literature review study is presented in section II, proposed solution for the security and QoS enhancement in the VANET is presented in section III, section IV presents the experimental study and presents comparative analysis to show the robust performance of proposed model. Finally, section V presents concluding remarks.

\section{LiTERATURE SURVEY}

This section represents brief discussion about recent techniques of secure communication in VANETs. This section includes various schemes such as authentication, key generation, key exchange, hash function and cryptography schemes. Ref. by [20] presented a robust approach for secure and QoS aware routing approach for VANET. According to this approach, Ant colony optimization scheme is used to find the optimal route based on the data traffic type. The ACO scheme helps to achieve the best fit solution for the given problem. Later, VANET-oriented Evolving Graph (VoEG) model is developed to measure the likelihood among vehicles. Ref. by[21] introduced 2FLIP approach to maintain the location privacy. This process uses message authentication code (MAC) and hash operations to induce the two-factor authentication. Moreover, this approach uses biometric system for each driver to collect the traces of each driver where these biometrics are verified using tamper-proof device (TPD) is embedded in on board unit (OBU). In order to secure the $\mathrm{V} 2 \mathrm{~V}$ and $\mathrm{V} 2 \mathrm{R}$ communication, one-way hash function are generated, the message is secured using MAC generation and a hash function is re-generated for verification. Ref. by [22] introduced an authentication model for anonymous users based on the signature and message recovery. This approaches uses batch operations to authenticate the multiple signature which helps to reduce the authentication time. The main contributions of approach are as follows: ID based anonymous signature scheme is developed for authentication where length of the packet are shorter, resulting in reduced communication overhead. In the next stage, the message is recovered using signature which reduces the computation overhead by neglecting the message with invalid signature. Finally, batch authentication is used where all the messages can be authentication at the same time. Bad mouthing and providing the false information are the serious issues in VANETs. Generally, reputation management schemes are used for this purpose but it cannot handle the self-promoting attack and it may violate location privacy. To deal with these issues, Ref. by [23] presented privacy preserving and reputation management model to mitigate the bad mouthing attacks. This work presents a service reputation which is used for computing the QoS of the user, if any user provides low QoS then it is identified as malicious node. Furthermore, this work focuses on the location privacy by presenting the hidden-zone and k-anonymity scheme. Ref. by [24] discussed that the current routing scheme do not ensure the on-time packet delivery due to high dynamic nature of VANETs which affects the process of safety alert message. These safety messages require security to maintain the hassle free traffic hence in this work a secure routing scheme VANSecis presented to avoid the threats to the network. This approach is based on the trust management which identifies the false and malicious nodes.

Ref. by [25] presented Ad hoc On-demand Multipath Distance Vector (AOMDV) routing algorithm. This algorithm provides minimum three paths to route the packets. However, AOMDV suffers from the lack of security scheme, cryptography and intrusion detection schemes because of these issues this protocol is vulnerable to the various threats such as black hole and 
man in the middle attack. Hence, this wok introduces secure and efficient AOMDV protocol for VANETs. The security is enabled by detecting the malicious vehicles which are not authenticated and pose malicious behaviour. Furthermore, best path is obtained using Route Reply (RREP) packet. Ref. by [26] focused on the data security in VANETs and suggested that the secured data can be delivered using LEACH protocol. Hence, in this work, authors considered the combination of LEACH protocol and lightweight cryptographic model. For increasing the security, the Random Firefly is used for identifying the trustworthy vehicles in the considered network topology. After identifying the reliable vehicles, the lightweight security and Hash function methods are used for securing the information for transmission. Ref. by [27] presented Security Aware Fuzzy Enhanced Reliable Ant Colony Optimization (SAFERACO) routing protocol to distinguish the malicious and trustworthy nodes during communication. The misbehaving nodes are discarded from the routing process. User authentication plays important role to improve the security of VANETs. Several approaches are present based on authentication.

\section{Proposed Model}

This section presents the proposed model for secure and efficient communication in vehicular Ad-Hoc networks. Significant amount of works have been carried out to improve the communication performance but security remains a challenging task. Moreover, the dynamic network topology creates several challenging issue. Thus, user authentication and key management becomes a tedious task to maintain the cure communication. This research work focus on the key management and data security. The proposed model of SGKC organized as follows:

A. First of all, we deploy a Vehicular Ad-Hoc network and define the preliminary and initial assumptions related to the network.

B. In the next phase, V2V, V2I and V2X communication protocol is presented where key management, authentication, key exchange modules are presented

C. Finally, the cryptography scheme is presented to secure the data packets.

\section{A. Preliminaries and Network Modelling}

The VANET architecture contains several components such as trusted authority (TA), road side units (RSUs), service provider (SP), and onboard unit (OBU) mounted vehicles as shown in figure 2. Each entity of network has assigned specific tasks. Generally, TA is considered as car manufacturer or transport management departments. Trust authority is responsible for registering the RSUs, generates the public and private keystoauthenticate each user. TA performs several computations hence we assume that enough storage is provided to TA along with adequate computation capability. Road Side Units (RSUs) are the infrastructures which are deployed at the road intersection and road side which act as relays for V2I communication. 


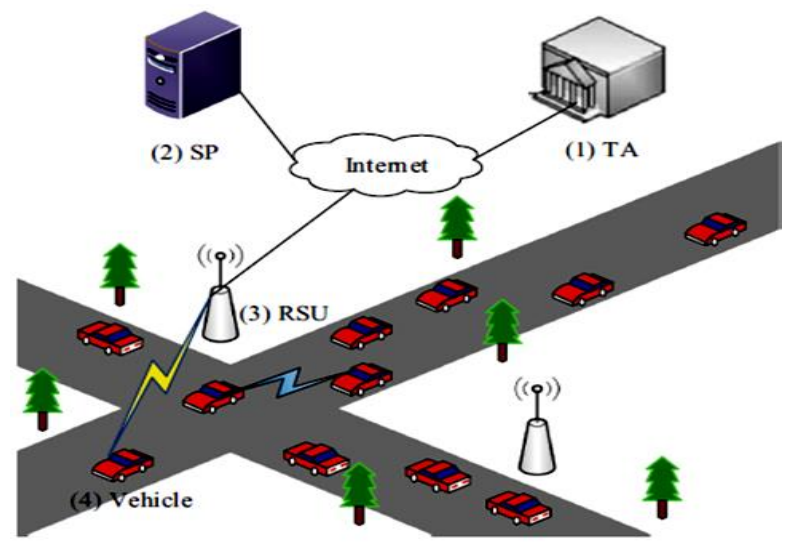

Fig. 2: VANET Model

The communication between RSU and vehicle is performed using dedicated short-range communications (DSRC) protocol. The main task of RSU is to verify the legitimacy of the received message from vehicles [8]. The Service provider provides different types of application to all vehicles. In order to provide the application services, the RSU receives the message from vehicles, verifies its legitimacy and if the message is valid then forwarded to the application server for providing the required service. The SA, TA and RSU can communicate through a safety cable channel. Similarly, OBU is a wireless unit which is installed on the vehicle with GPS and a small device for short range communications.

\section{B. Security Requirements}

In VAETs, data security and privacy are considered as important factor to develop the secure VANET model. This work focuses on the following security requirements [28][29][30]:

- User authentication and message integrity: in this architecture, once the message is transmitted to the receiver, then the receiver must ensure the message integrity and validity by verifying the signatures.

- Vehicle identity protection: the actual vehicle identity is only known by the trusted authority and the vehicles. This helps to maintain the anonymity from other vehicles in the network.

- Message traceability: during communication, if any bogus message is received by the receiver, then TA should be able to track the original identity of the vehicle.

- Message stealing: during data communication of message transmission phase, the protocol should be able the secure the high confidentiality message by avoiding the message stealing by attackers.

- Fake Message attack: the fake messages are disseminated to harm the network entities hence the protocol should restrict the fake message circulation in the network.

- Fake identity: according to this attack the real identity of vehicle is forged and used for concealing the information. Hence, the identity of vehicles should be anonymized to prevent this attack.

Similarly, here it focus on achieving the solution for non-repudiation attack, replay attack and DOS attack to develop a more robust and secure network architecture.

Hence, in this work introduced a combined novel key management and data security approach for VANETs. The proposed model is implemented in two-fold manner where first of all key 
management is performed and later, data encryption is applied. The proposed approach is denoted as SGKC (Secure Group Key Management and Cryptography).

Table.1: Mathematical Properties

\begin{tabular}{|c|l|}
\hline $\mathcal{G}_{o}, \mathcal{G}_{N}$ & Additive cyclic group \\
\hline$p$ & Large prime order for additive cyclic group \\
\hline $\mathrm{F}$ & Bilinear map function \\
\hline $\mathcal{R}_{R S U}$ & Random key for RSU \\
\hline$K_{R S U}$ & Public key of RSU \\
\hline $\mathbb{H}$ & Hash function \\
\hline
\end{tabular}

\section{SGKC (Secure Group Key Management and Cryptography).}

\section{(I) Group Key Management}

This work first describes the bilinear map generation to incorporate the secure functionality in the network. Let us consider that $\mathcal{G}_{o}$ and $\mathcal{G}_{N}$ are the additive cyclic group with the large prime order $p$. A map function $\mathrm{F}$ is computed as in equation 1

$$
\widehat{\mathrm{F}}: \mathcal{G}_{o} \times \mathcal{G}_{N} \rightarrow \mathcal{G}_{N}
$$

In which it should satisfy the following conditions to generate the bilinear pairing.

- Bilinear: for all $M, N \in \mathcal{G}_{o}$ and for all $a, b \in Z_{p p}^{*}$, the function is $\hat{\mathrm{F}}(a M, b N)=$ $\hat{\mathrm{F}}(a M, b N)^{a b}$. Similarly, for all $M, N, Y \in \mathcal{G}_{o}$ the bilinear map as in equation 2 and 3.

$$
\begin{aligned}
\hat{\mathrm{F}}(M+N, Y) & =\hat{\mathrm{F}}(M, Y) \hat{\mathrm{F}}(N, Y) \\
\hat{\mathrm{F}}(M, N+Y) & =\hat{\mathrm{F}}(M, N) \hat{\mathrm{F}}(M, Y)
\end{aligned}
$$

- Non-degenerate: there exists that the $M, N \in \mathcal{G}_{o}$, there is $\widehat{\mathrm{F}}(M+N, Y) \neq 1$

- Computability: for all $M, N \in \mathcal{G}_{o}$ efficient approach is present to compute the $\widehat{\mathrm{F}}(M, N)$

- Symmetric: As per equation $\mathrm{C} 4$ for all $M, N \in \mathcal{G}_{o}$

$$
\widehat{\mathrm{F}}(M, N)=\widehat{\mathrm{F}}(N, M)
$$

According to the proposed approach, first it represents the authentication process between RSU and vehicle. The complete authentication process is divided into three phases as initialization, authentication and distribution of group keys. The working process of these stages is described in the following subsections.

\section{I.a. Initialization}

In this first step of proposed SGKC approach, we perform user registration and key allocation for each vehicle in the network. In VANET, vehicle must be registered with the TA then TA assigns secret information to the corresponding vehicle. During this process, the TA stores driver's information such as contact information, address, and licence plate number. Let us consider that the $G_{\mathcal{H}}$ as cyclic additive group, $Q_{\mathcal{H}}$ is the generator and unique vehicle id is denoted as $i d$. Here, we adopt the Hash function as $k:\{0,1\}^{*} \times G_{\mathcal{H}} \rightarrow Z_{p}^{*}$ where $Z_{p}^{*}$ denotes the nonnegative integer 
set which is less than the prime number $p$. Based on these assumptions, the TA generates a secret key $S_{i d}$ for each vehicle in the network. The key is given as in equation 5.

$$
S_{i d}=h\left(i d, Q_{\mathcal{H}}\right)
$$

The generated key is assigned to the appropriate vehicle after registration. The secret key for each user/vehicle is stored in the TA's key storage dataset. Simultaneously, the TA selects a random integer to assign the private key for RSU. This random number is selected as $\mathcal{R}_{R S U} \in Z_{p}^{*}$. Let $G_{1}$ be an additive cyclic group of order $q$ generated by $P$. Thus, the RSU public key can be computed as in equation 6.

$$
K_{R S U}=\mathcal{R}_{R S U} P
$$

Here, RSU public key, generator $P$, hash function $k$ and $G_{1}$ will be published to all devices whereas the RSU private secret key $\mathcal{R}_{R S U}$ is kept secret during this process. This process is used for registering the vehicle. Let us assume that the registered vehicle is entering the range of RSU. If that vehicle demands for any service from the VANET, then key assignment is the necessary task. This vehicle $v$ selects a partial private key as $R_{v} \in Z_{p}^{*}$, and the corresponding partial public key is given as in equation 7 .

$$
Q_{v}=\mathcal{R}_{v} P
$$

Where $P$ is the generator, using these parameters service request, public key and vehicle id are delivered to the corresponding RSU which are arranged as $\left\langle\right.$ Service Request, $\left.Q_{v}, i d\right\rangle$. once the partial public key $Q_{v}$ is generated, the RSU request to TA for providing the secret key $S$ for vehicle $i d$ i.e. RSU request to TA for $S_{i d}$. At this stage, we generate a secure hash function as $\mathbb{H}:\{0,1\}^{*} \times G_{1} \rightarrow G_{1}$. With the help of this, the partial keys can be generated as in equation 8 .

$$
Q_{i d}=\mathbb{H}\left(i d, Q_{R S U}\right)
$$

Based on the secret key, partial public key and secret key of RSU, a certificate is delivered to the vehicle as in equation 9 .

$$
C=Q_{i d} S_{i d} \mathcal{R}_{R S U}
$$

Thus, the partial private key can be derived as in equation 10 .

$$
R_{u}=R_{R S U} Q_{i d}
$$

Now, the public key can be presented as $\left\langle Q_{v}, i d\right\rangle$ and the private key set is given as $\left\langle\mathcal{R}_{v}, \mathcal{R}_{u}\right\rangle$

\section{I.b. Authentication}

In this process, we present authentication process for the vehicle. We assume that at a time $t$, the vehicle starts using the road message service. The partial public key and time combine as in equation 11 . 


$$
Q_{1}=Q_{v} t=\mathcal{R}_{v} P t
$$

Moreover, a cyclic group $G_{2}$ is generated with the prime order $s$ and the bilinear operator is given as $\hat{\mathrm{F}}: G_{1} \times G_{1} \rightarrow G_{2}$. Here, the intermediate value of partial public key can be obtained as in equation 12 .

$$
Q_{i d}=\mathbb{H}\left(i d, Q_{R S U}\right)
$$

whereid is the vehicle id, and $Q_{R S U}$ is the public key of RSU which are already known to the vehicle. Along with this, we generate two important parameters $\alpha$ and $\beta$ for authentication as in equation 13.

$$
\begin{aligned}
& \alpha=\hat{\mathrm{F}}\left(Q_{R S U}, Q_{i d}\right) \\
& \beta=h\left(t \| \alpha, \mathcal{R}_{i d}\right)
\end{aligned}
$$

Where $\mathcal{R}_{i d}$ is the secret key of vehicle which is allocated during initialization phase. Based on these parameters, we generate the final signature as in equation 14 .

$$
U=\mathcal{R}_{u}+Q_{i d} \mathcal{R}_{v} t v
$$

From here, the vehicle sends the authentication request as $\langle U, i d, t, v\rangle$ and RSU performs the verification process whether $\alpha=\frac{\hat{\mathrm{F}}(P, U)}{\hat{\mathrm{F}}\left(Q_{1}, Q_{i d}\right)^{v}}$. In order to deliver the message, the following verification condition must be satisfied as in equation 15 .

$$
\frac{\widehat{\mathrm{F}}(P, U)}{\widehat{\mathrm{F}}\left(Q_{1}, Q_{i d}\right)^{v}}=\hat{\mathrm{F}}\left(P, Q_{i d}, \mathcal{R}_{R S U}\right)=\hat{\mathrm{F}}\left(\mathcal{R}_{R S U}, P, Q_{i d}\right)=\hat{\mathrm{F}}\left(\mathcal{R}_{R S U}, Q_{i d}\right)
$$

After satisfying this condition, the authentication phase is completed.

\section{I.c. Key Distribution}

In this phase, the generated group keys are distributed to each legitimate vehicle. This key assignment is done by TA. Let us assume that the secret $\varepsilon \in Z_{p}^{*}$ is randomly selected by TA, and then RSU computes as in equation 16.

$$
\begin{gathered}
\mathbb{W}=\mathcal{E} Q_{v} T \\
\mathbb{F}=h\left(\mathbb{W} \| v, \mathcal{R}_{u}\right)
\end{gathered}
$$

Here RSU is capable to generate the partial public $\mathcal{R}_{u}$ as described before. Now,the $\langle\mathbb{W}, \mathbb{F}, T\rangle$ iscomputed by RSU and transmitted to vehicle. Here our aim is to combine the secret key with the current time stamp $T$. In this process, the vehicle compares the value of $F$ with stored values and if it is found valid then secret is derived as in equation 17. 


$$
\begin{gathered}
N=\mathbb{W} T^{-1} \mathcal{R}_{v}^{-1}=Q_{v} T \mathcal{E} T^{-1} \mathcal{R}_{v}^{-1} \\
=\mathcal{E} P
\end{gathered}
$$

Hence, the final group can be achieved as in equation 18 .

$$
G_{k}=h(N)=h(\varepsilon p)
$$

\section{(II) Data encryption and decryption}

This phase presents the data encryption and decryption approach to provide the secure data exchange. According to this process, the first task is to secure the data using encryption key which is used by receiver to encrypt and decrypt the data by sender and receiver. This phase uses the state value(state) of receiver vehicle as encryption key. In order to maintain the location privacy, methodology uses hash the state value before transmitting to the corresponding vehicle.

\section{II.a. Key Generation}

This complete process of data encryption and process of key generation is shown in figure 3.

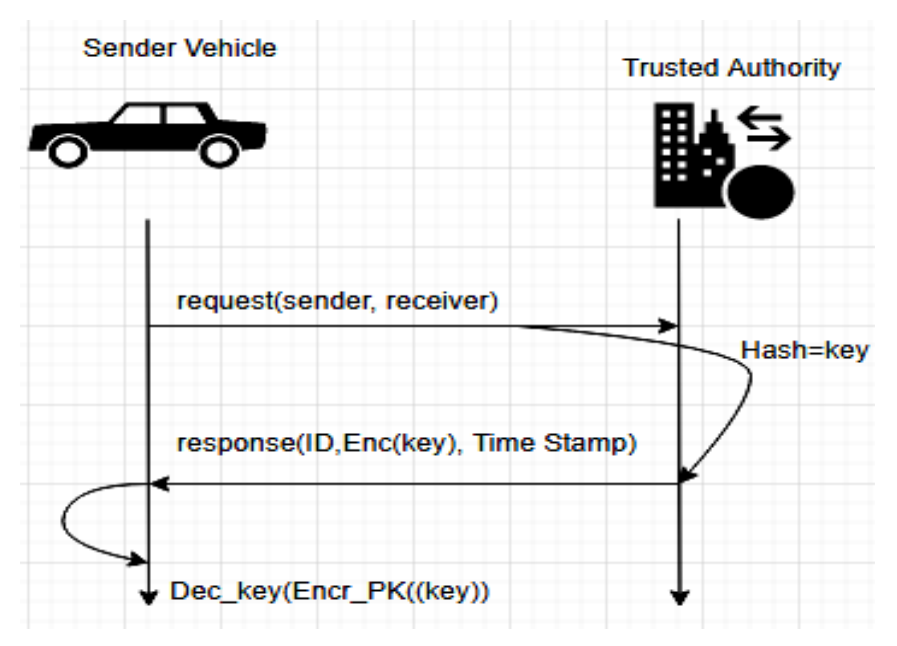

Fig. 3: Data encryption process and key generation

As shown in Figure 3, complete process of key generation is completed by using following steps:

(a) The sending vehicle $v_{s}$ sends the message request (Message req $_{\text {) }}$ ) of receiver vehicle $v_{r}$ request to the trusted authority where public key is used for encrypting the message request. This is expressed as in equation 19.

$$
\operatorname{Cipher}_{V_{S} T A}=\operatorname{Encr}_{T A_{p k}}\left(\mathbb{S}_{I d}+\mathbb{R}_{I D}\right)
$$

As given in eq. 15 , the public key of TA is used for encrypting the sender vehicle id $\mathbb{S}_{I d}$ and receiver vehicle $\mathbb{R}_{I D}$

(b) The trusted authority decrypts the cipher text of eq. (15) using its own secret key as in equation 20. 


$$
\operatorname{Dec}_{\text {Message }}=\operatorname{Decr}_{T A_{s k}}\left(\text { Cipher }_{V_{S} T A}\right)
$$

This decryption process provides the state values of receiver vehicle and hashes these values. Hash values are considered as the key for sender vehicle to encrypt the data. This is denoted as in equation 21 .

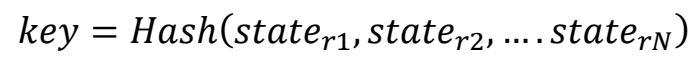

(c) TA uses sender public key to encrypt the key, the encrypted data and time stamps are send to the sender vehicles from TA. The final received message from TA is denoted as in equation 22 .

$$
\operatorname{Cipher}_{T A V_{s}}=\operatorname{Encr}_{V s_{p k}}(I D+\text { key }+ \text { Timestamp })
$$

(d) After receiving the data from (18) sender vehicle uses own private key to decrypt this data and gets the real key for further encryption along with the time stamps. This is computed as in equation 23 .

$$
\text { Timestamp }+ \text { key }=\operatorname{Decr}_{V s_{p k}}\left(\text { Cipher }_{T A V_{s}}\right)
$$

\section{II.b. Data Encryption \& Decryption}

Before transmitting the data from sender vehicle to receiver vehicle we encrypt the data using time stamp and secret key to provide the data security during transmission. This encryption format is given as in equation 24 .

$$
\text { Message }=\text { Timestamp }+\mathbb{R}_{I D}+\text { Encr }_{\text {key }}(I D+\text { data })
$$

This encrypted data is transmitted to the receiver vehicle where data decryption is performed through repeal mechanism.

\section{RESUltS AND DISCUSSION}

This section shows the experimental analysis using proposed approach. The obtained performance is compared with the existing techniques. This research work focused on ensuring the security for VANET.

\subsection{Achieved Security Issues}

These proposed works achieve the following security issues such as:

- Authentication: In this work, authentication is an important task to avoid the attacker nodes to join the network. Later, Hash values are obtained from the key and authentication process is performed after achieving the RREP message from the communicating node.

- Message confidentiality: This work applies symmetric cryptography where public and private secrete keys are generated from the RSA key generation method.

- Location privacy and anonymity: This security aspect is obtained by generating the Hash of the location of the vehicle and vehicle ID. 


\subsection{Performance Measurement Parameters}

This section presents the experimental analysis using proposed approach. The performance of proposed approach is measured in terms of packet loss, throughput, packet deliver, end-to-end delay, average message delay, and message loss ratio. The simulation parameters are given in table 2.

Table.2: Simulation Parameters

\begin{tabular}{|l|l|}
\hline Simulation Parameter & Used Value \\
\hline Simulation Area & $1500 \mathrm{~m} \times 1500 \mathrm{~m}$ \\
\hline Simulation Time & $100 \mathrm{~s}$ \\
\hline Data Traffic & CBR \\
\hline Route protocol & AODV \\
\hline Mobility & Random Waypoint \\
\hline Channel bandwidth & $6 \mathrm{Mbps}$ \\
\hline
\end{tabular}

According to the table 2, proposed approach considered total 100 nodes which are deployed in the $1500 \mathrm{~m} \times 1500 \mathrm{~m}$ area. The vehicles follow the Random Waypoint model with the constant bit rate data traffic. Total 10 nodes are considered as faulty node which is responsible for various attacks such as Denial-of-service, black hole, and badmouthing etc. In this work, we measure the performance of proposed approach under various attacks to show the robust performance. The obtained performance is measured using following performance metrics:

(a) Packet Loss Ratio: is measured by taking the ratio of the dropped packets which are generated from the source but not delivered to the destination as in equation 25 .

$$
P L R=\frac{P_{\text {Sent }}-P_{\text {received }}}{P_{\text {Sent }}} \times 100
$$

Where $P_{\text {Sent }}$ denotes the number of sent data packets, $P_{\text {received }}$ denotes the received number of data packets.

(b) Throughput: is measured by computing the total of bytes received successfully in one communication session. This is computed as in equation 26.

$$
\text { Throughput }=\frac{P_{\text {Sent }}-P_{\text {received }}}{P_{\text {Sent }}} \times 100
$$

(c) Packet delivery ratio: this is measured by taking the ratio of delivered packet to the destination which are generated from source nodes. It can be calculated as in equation 27.

$$
P D R=\frac{P_{\text {received }}}{P_{\text {Sent }}} \times 100
$$

(d) Average end-to-end delay: this is the time take by the data packet to reach to the destination. During this phase, the route discovery, data retransmission and propagation time etc. are considered. This is computed as in equation 28. 


$$
\text { Delay }=\frac{\sum_{i=1}^{P_{\text {succes }}}\left(D_{i}-s_{i}\right)}{P_{\text {Succes }}} \times 100
$$

Where $D_{i}$ denotes the $i^{\text {th }}$ packet receiving time, $s_{i}$ denotes the sending time for $i^{t h}$ packet and $P_{\text {succes }}$ denotes the number of successfully transmitted packets.

(e) Average message delay: this is the measurement of total delay occurred to deliver the message from one source to destination. This can be computed as in equation 29.

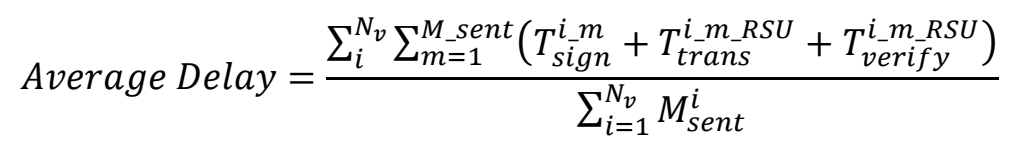

Where $N_{v}$ is the total number of vehicles, $M_{\text {sent }}^{i}$ is the total number of packet sent by vehicle $i, T_{\text {sign }}^{i-m}$ is the time required to sign a message by vehicle, $T_{\text {trans }}^{i_{-} m_{2} R S U}$ is the time require to transmit the message $m$ to RSU and $T_{\text {verify }}^{i \text { m } R S U}$ is the time required for authentication. Similarly, we measure the message loss ratio asin equation 30 .

$$
\text { Message lossr atio }=\frac{\sum_{i=1}^{N_{v}} M_{\text {sent }}^{i}-\sum_{r=1}^{R S U^{n}} M_{r e c}^{r}}{R S U^{n} * \sum_{i=1}^{N_{v}} M_{\text {sent }}^{i}}
$$

\subsection{Comparative Performance Analysis}

This section shows the comparative experimental analysis where performance of proposed approach is compared with the existing techniques by varying the number of vehicles, speed and malicious nodes in the network.

\section{(a) Varying Vehicles and Fixed Speed}

In this phase, performance is evaluated by varying the number of vehicles ranging from 20 to 100 with 10 numbers of malicious nodes present in the network and the speed of vehicles is fixed in the range of 70-72kmph. First it computes the packet loss ratio for this experimental setup and compared the performance with AOMDV [25] and SE-AOMDV [25] protocols. Figure 5 shows a comparative performance in terms of packet loss ratio. According to this experiment, the existing protocols AOMDV [25] and SE-AOMDV [25] [25] drop the packet due to malicious nodes in the network. However, the existing protocols suffer from the malicious nodes and drop the packets whereas proposed approach shows robust performance. The average packet loss rate is obtained as $1.26 \%, 1.68 \%$ and $0.84 \%$ using AOMDV [25], SE-AOMDV [25], and Proposed approach. This experiment shows that the proposed approach achieves $0.66 \%$ and $0.49 \%$ improvement when compared with the AOMDV [25] and SE-AOMDV [25] methods. 


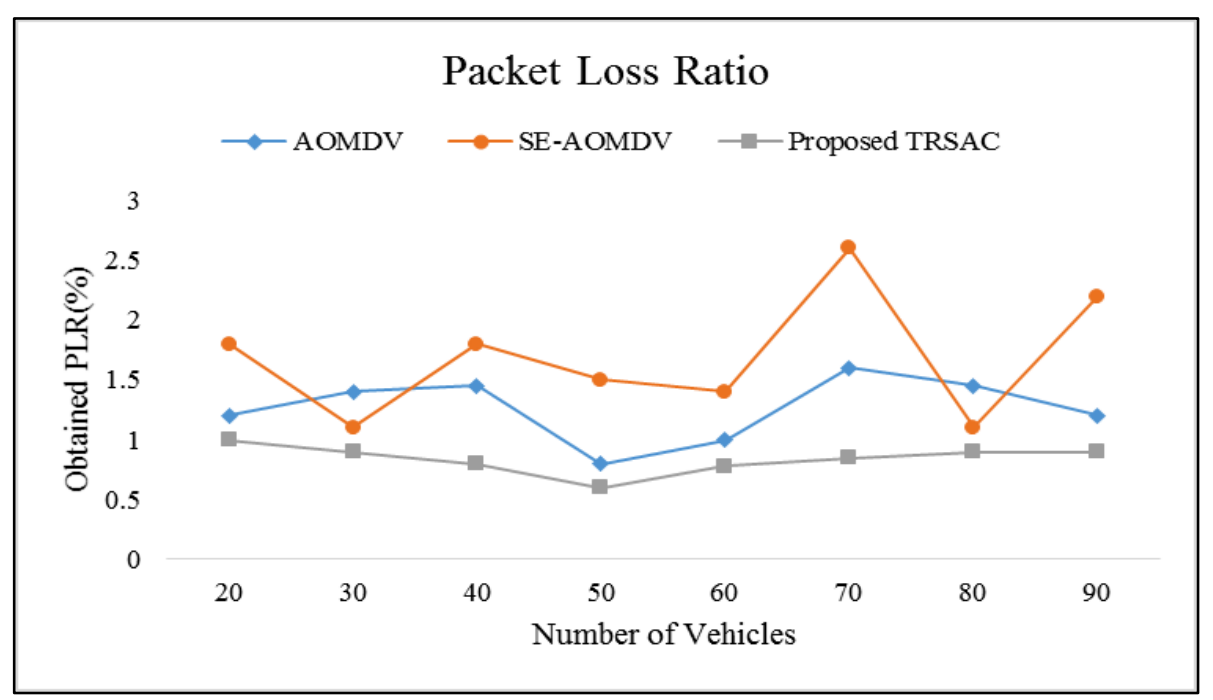

Fig. 5: Packet Loss ratio performance

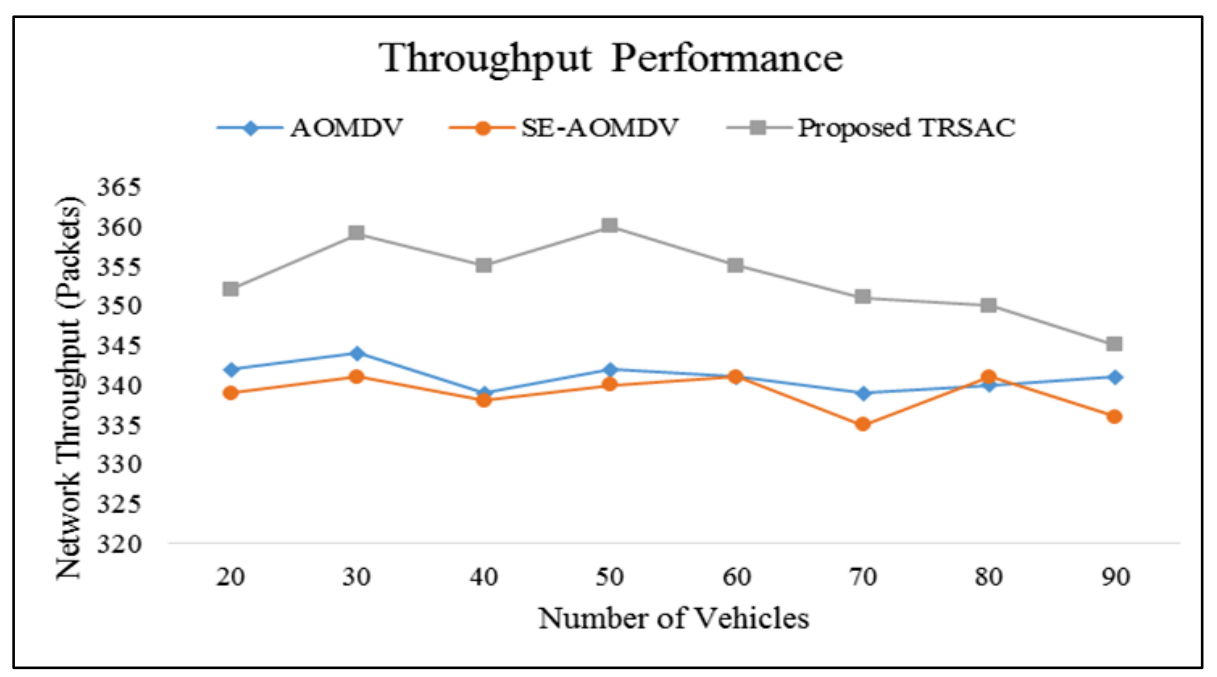

Fig. 6: Througput performance

In next phase of the proposed approach, it measures the throughput performance for same experiment setup. The obtained performance is depicted in figure 6 . The more number of vehicles creates issues in link stability and frequent selection of relay nodes creates a complex environment for communication leading towards the decreased throughput, whereas proposed approach helps to main the network reliability and reduces packet drops. The average network throughput performance is reported as 341, 338.875 and 353.375 using AOMDV [25], SEAOMDV [25] and proposed approach. Similarly, we compute the end-end delay performance for varied number of vehicles for the considered experimental scenario. The obtained performance is depicted in figure 7. This experiment shows that the average end-to-end delay is obtained as 4.28ms, 1.56ms, and 1.1ms using AOMDV [25], SE-AOMDV [25] and proposed approach. 


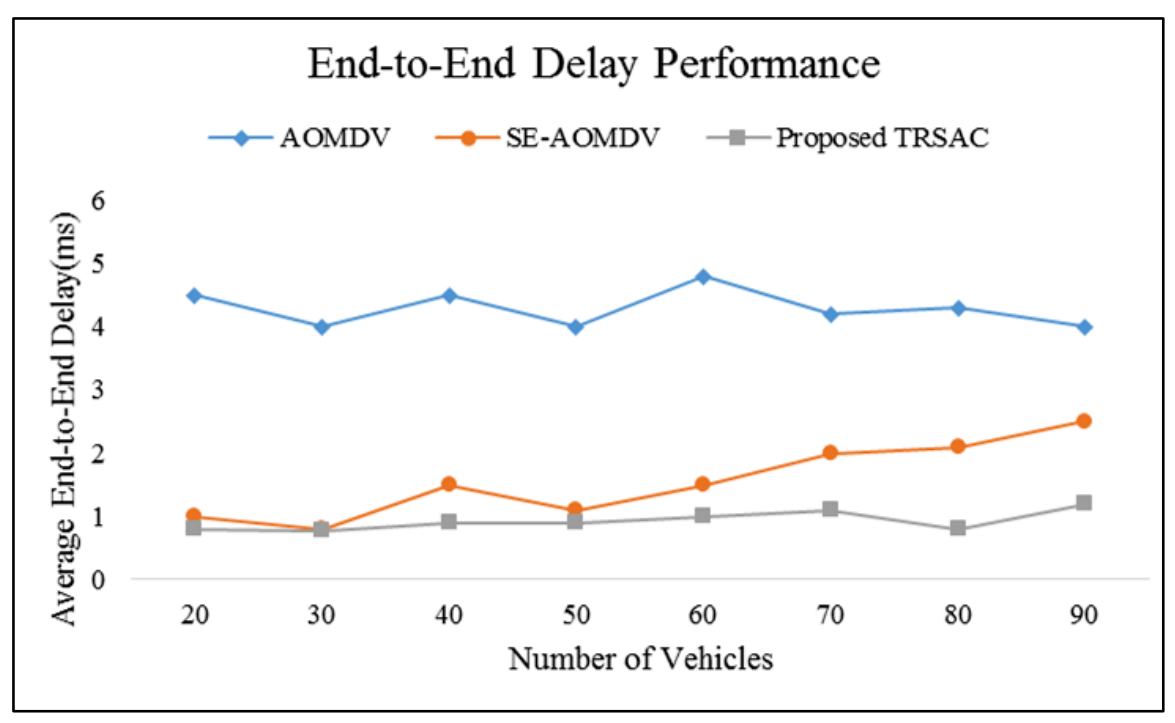

Fig. 7: End-to-End delay performance

\section{Conclusion}

This proposed approach focused on improving the VANET by incorporating key management and data cryptography process. According to proposed approach, first it introduces a novel key management scheme where any new upcoming vehicle is registered with Trusted Authority (TA) and authenticated to perform the communication. This helps to maintain the security and reduces outsider attacks. In the next phase, introduced Elliptic curve cryptography scheme to encrypt and decrypt the data during vehicle communication. Hence, the proposed approach provides better security. Moreover, the proposed approach uses lightweight computations which help to reduce the computational overhead of the network. The comparative study is carried out which shows the improved performance using proposed approach.

\section{REFERENCES}

[1] Liu, J., Wan, J., Wang, Q., Deng, P., Zhou, K., \& Qiao, Y. (2015). A survey on position-based routing for vehicular ad hoc networks. Telecommunication Systems, 62(1), 15-30. doi:10.1007/s11235-015-9979-7.

[2] Tomar, R., Prateek, M., \& Sastry, G. H. (2016). Vehicular adhoc network (VANET)-an introduction. International Journal of Control Theory and Applications, 9(18), 8883-8888.

[3] Zhang, W., Xiao, X., Wang, J., \& Lu, P. (2018, November). An improved AODV routing protocol based on social relationship mining for VANET. In Proceedings of the 4th International Conference on Communication and Information Processing (pp. 217-221). ACM.

[4] Yang, X., Sun, Z., Miao, Y., Wang, N., Kang, S., Wang, Y., \& Yang, Y. (2015, March). Performance Optimisation for DSDV in VANETs. In 2015 17th UKSim-AMSS International Conference on Modelling and Simulation (UKSim) (pp. 514-519). IEEE.

[5] Abdelgadir, M., Saeed, R. A., \& Babiker, A. (2017). Mobility routing model for vehicular Ad-Hoc networks (VANETS), smart city scenarios. Vehicular Communications, 9, 154-161.

[6] Kadadha, M., Otrok, H., Barada, H., Al-Qutayri, M., \& Al-Hammadi, Y. (2017, June). A streetcentric QoS-OLSR protocol for urban vehicular ad hoc networks. In 2017 13th International Wireless Communications and Mobile Computing Conference (IWCMC) (pp. 1477-1482). IEEE.

[7] Silva, R., Lopes, H. S., \& Godoy, W. (2013, September). A heuristic algorithm based on ant colony optimization for multi-objective routing in vehicle ad hoc networks. In 2013 BRICS Congress on Computational Intelligence and 11th Brazilian Congress on Computational Intelligence (pp. 435440). IEEE. 
[8] Taherkhani, N., \& Pierre, S. (2012, October). Congestion control in vehicular ad hoc networks using meta-heuristic techniques. In Proceedings of the second ACM international symposium on Design and analysis of intelligent vehicular networks and applications (pp. 47-54). ACM.

[9] Aadil, F., Bajwa, K. B., Khan, S., Chaudary, N. M., \& Akram, A. (2016). CACONET: ant colony optimization (ACO) based clustering algorithm for VANET. PloS one, 11(5), e0154080.

[10] Jindal, V., \&Bedi, P. (2018). An improved hybrid ant particle optimization (IHAPO) algorithm for reducing travel time in VANETs. Applied Soft Computing, 64, 526-535.

[11] Li, G., Ma, M., Liu, C., \& Shu, Y. (2017). Adaptive fuzzy multiple attribute decision routing in VANETs. International Journal of Communication Systems, 30(4), e3014.

[12] Bagherlou, H., \& Ghaffari, A. (2018). A routing protocol for vehicular ad hoc networks using simulated annealing algorithm and neural networks. The Journal of Supercomputing, 1-25.

[13] Sampigethaya, K., Huang, L., Li, M., Poovendran, R., Matsuura, K., \&Sezaki, K. (2005). CARAVAN: Providing location privacy for VANET. Washington Univ Seattle Dept of Electrical Engineering.

[14] Sampigethaya, K., Li, M., Huang, L., \&Poovendran, R. (2007). AMOEBA: Robust location privacy scheme for VANET. IEEE Journal on Selected Areas in communications, 25(8), 1569-1589.

[15] Wasef, A., \& Shen, X. S. (2010). REP: Location privacy for VANETs using random encryption periods. Mobile Networks and Applications, 15(1), 172-185.

[16] Chim, T. W., Yiu, S. M., Hui, L. C., \& Li, V. O. (2012). VSPN: VANET-based secure and privacypreserving navigation. IEEE transactions on computers, 63(2), 510-524.

[17] Rahbari, M., \& Jamali, M. A. J. (2011). Efficient detection of Sybil attack based on cryptography in VANET. arXiv preprint arXiv:1112.2257.

[18] Mejri, M. N., Achir, N., \& Hamdi, M. (2016, January). A new group Diffie-Hellman key generation proposal for secure VANET communications. In 2016 13th IEEE Annual Consumer Communications \& Networking Conference (CCNC) (pp. 992-995). IEEE.

[19] Lu, H., Li, J., \& Guizani, M. (2012, January). A novel ID-based authentication framework with adaptive privacy preservation for VANETs. In 2012 Computing, Communications and Applications Conference (pp. 345-350). IEEE.

[20] Eiza, M. H., Owens, T., \& Ni, Q. (2015). Secure and robust multi-constrained QoS aware routing algorithm for VANETs. IEEE Transactions on Dependable and Secure Computing, 13(1), 32-45.

[21] Wang, F., Xu, Y., Zhang, H., Zhang, Y., \& Zhu, L. (2015). 2FLIP: A two-factor lightweight privacypreserving authentication scheme for VANET. IEEE Transactions on Vehicular Technology, 65(2), 896-911.

[22] Liu, Y., He, Z., Zhao, S., \& Wang, L. (2016). An efficient anonymous authentication protocol using batch operations for VANETs. Multimedia Tools and Applications, 75(24), 17689-17709.

[23] Wang, J., Zhang, Y., Wang, Y., \& Gu, X. (2016). RPRep: A robust and privacy-preserving reputation management scheme for pseudonym-enabled VANETs. International Journal of Distributed Sensor Networks, 12(3), 6138251.

[24] Ahmed, S., Rehman, M. U., Ishtiaq, A., Khan, S., Ali, A., \& Begum, S. (2018). VANSec: Attackresistant VANET security algorithm in terms of trust computation error and normalized routing overhead. Journal of Sensors, 2018.

[25] Makhlouf, A. M., \&Guizani, M. (2019). SE-AOMDV: secure and efficient AOMDV routing protocol for vehicular communications. International Journal of Information Security, 1-12.

[26] Manickam, P., Shankar, K., Perumal, E., Ilayaraja, M., \& Kumar, K. S. (2019). Secure Data Transmission Through Reliable Vehicles in VANET Using Optimal Lightweight Cryptography. In Cybersecurity and Secure Information Systems (pp. 193-204). Springer, Cham.

[27] Zhang, H., Bochem, A., Sun, X., \&Hogrefe, D. (2018, June). A security aware fuzzy enhanced reliable ant colony optimization routing in vehicular ad hoc networks. In 2018 IEEE Intelligent Vehicles Symposium (IV) (pp. 1071-1078). IEEE.

[28] Mr. MahabaleshwarKabbur \& Dr. V. Arul KumarS, "Cooperative RSU Based Detection and Prevention of Sybil Attacks in Routing Process of VANET" in 2020, IOP Publishing conference series J. Phys.: Conf. Ser. 1427012009

[29] Mr. MahabaleshwarKabbur \& Dr. V. Arul KumarS, "Detection and Prevention of DoS Attacks in VANET with RSU's Cooperative Message Temporal Signature” in July 2019 ISSN: 2277-3878, Volume-8 Issue-2. 
[30] Mr. MahabaleshwarKabbur \& Dr. V. Arul KumarS, "MAR_Worm: Secure and Efficient Wormhole Detection Scheme through Trusted Neighbour Nodes in VANETs" in December 2019 ISSN: $2278-$ 3075, Volume-9 Issue-2S.

\section{AUTHORS}

Mr. Mahabaleshwar Kabbur, research scholar of REVA University. He has obtained his Master's degree in Computer Applications (MCA) and research degree in Master of Philosophy in computer science (M.Phil). He has 14 years of experience in teaching and 03 years of experience in research. He is pursuing his doctoral research on "Security on Wireless networking with respect to VANET". He is published 12 research articles in UGC approved international journals and presented 15 articles in various National and International conferences. His specializations and research interests include Network Security, Content-Based Image Retrieval Techniques \&IoT.

Dr. V. Arul Kumar, Assistant Professor in School of Computer Science \& Applications REVA University holds doctoral degree in Computer Science from Bharathidasan University-Tamil Nadu. He has completed B. Sc (Applied Sciences - Computer Technology), M.Sc (Applied Sciences - Information Technology) from K.S.R College of Technology and M.Phil in Computer Science from Bharathidasan University, Tamil Nadu. He has 6 Years of experience in teaching and 8 years of experience in research. He has qualified in State Eligibility Test (SET) conducted by Mother Teresa Women's University. He has published 24 research articles in the various International / National

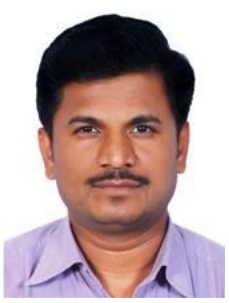
Journals and conferences. His Research area includes data Mining, cloud security and cryptography.

(C) 2020 By AIRCC Publishing Corporation. This article is published under the Creative Commons Attribution (CC BY) license. 